\title{
Development and Examination of the Social Appearance Anxiety Scale
}

\author{
Trevor A. Hart \\ David B. Flora
}

York University

\section{Sarah A. Palyo}

University of Buffalo (SUNY)

\section{David M. Fresco}

Kent State University

\section{Christian Holle}

William Paterson University

\section{Richard G. Heimberg}

Temple University

\begin{abstract}
The Social Appearance Anxiety Scale (SAAS) was created to measure anxiety about being negatively evaluated by others because of one's overall appearance, including body shape. This study examined the psychometric properties of the SAAS in three large samples of undergraduate students (respective $\mathrm{n} s=512,853$, and 541). The SAAS demonstrated a unifactorial structure with high test-retest reliability and internal consistency. The SAAS was positively associated with measures of social anxiety. The SAAS was also related to greater disparity between perceived, actual, and ideal physical attributes, beliefs that one's appearance is inherently flawed and socially unacceptable and that being unattractive is socially deleterious, feelings of unattractiveness, emphasis on appearance and its maintenance, and a preoccupation with being overweight. It was a unique predictor of social anxiety above and beyond negative body image indicators. Findings suggest that the SAAS is a psychometrically valid measure of social anxiety regarding one's overall appearance.
\end{abstract}

Keywords: social anxiety; body image; appearance; psychometric properties; measurement

Portions of this article were presented at the annual meeting of the Anxiety Disorders Association of America, Washington, D.C., March 2000, and the annual meeting of the Association for Advancement of Behavior Therapy, Philadelphia, PA, November 2001. The authors thank the research staff at the Adult Anxiety Clinic of Temple University for their help with data collection. Correspondence, requests for reprints, and requests for the SAAS should be sent to Trevor A. Hart, Department of Psychology, Ryerson University, Jorgenson Hall, 8th Floor, 350 Victoria Street, Toronto, ON, M5B 2K3 Canada; e-mail: trevor.hart@psych .ryerson.ca

Assessment, Volume 15, No. 1, March 2008 48-59

DOI: $10.1177 / 1073191107306673$

(C) 2008 Sage Publications 
Social anxiety disorder (also known as social phobia) is characterized by significant and persistent fear of social or performance situations in which embarrassment or humiliation may occur (Diagnostic and Statistical Manual of Mental Disorders [4th ed.], text revision; American Psychiatric Association, 2000). Individuals with social anxiety disorder report low quality of life, as well as substantial impairment in social, occupational, and educational functioning (Safren, Heimberg, Brown, \& Holle, 1997; Schneier et al., 1994). Holt, Heimberg, Hope, and Liebowitz (1992) identified four main classes of feared situations among patients with social anxiety disorder: formal speaking/interaction, informal speaking/interaction, observation by others, and assertion. These domains of feared situations are featured in a number of social anxiety measures, including the Liebowitz Social Anxiety Scale (Liebowitz, 1987), the Social Interaction Anxiety Scale (SIAS) and Social Phobia Scale (SPS; Mattick \& Clarke, 1998), the Social Phobia and Anxiety Inventory (SPAI; Turner, Beidel, Dancu, \& Stanley, 1989), and the Interaction Anxiousness and Audience Anxiousness scales (Leary, 1983b). However, other possible domains of concern to persons with social anxiety disorder have been less thoroughly examined. A specific situational fear that has received little research attention is that of fear of negative evaluation of one's appearance.

Fear of being negatively evaluated for one's appearance may also be highly relevant in the understanding of bulimia nervosa, anorexia nervosa, and body dysmorphic disorder. Each of these disorders is not only associated with body image disturbance but is also often associated with higher social anxiety and/or social impairment (American Psychiatric Association, 2000). Associations between social anxiety and maladaptive appearance schemas and body image disturbance have been found in both normative samples and clinical samples of individuals with eating disorders or body dysmorphic disorder (e.g., Cash \& Labarge, 1996; Coles et al., 2006; Hinrichsen, Waller, \& van Gerko, 2004; Pinto \& Phillips, 2005). Given the overlap between social anxiety and negative body image, there may be some usage in having a measure that taps into both constructs.

E. Hart, Leary, and Rejeski (1989) created the Social Physique Anxiety Scale (SPAS) to assess negative thoughts about one's appearance and discomfort during a physique evaluation. The items in this measure refer specifically to concerns about one's body form and structure (e.g., body fat, muscle tone, and general body proportions). Scores on the SPAS were positively correlated with social interaction anxiety and fear of negative evaluation in nonclinical samples and has been conceptualized as a subset of social anxiety (Frederick \& Morrison, 1998; E. Hart et al., 1989). SPAS scores have also been positively associated with body image dissatisfaction and depression (e.g., Diehl, Johnson, Rogers, \& Petrie, 1998; Frederick \& Morrison, 1998). Although the SPAS assesses concerns about the negative evaluation of one's appearance, one potential limitation is its exclusive focus on physique instead of appearance concerns more broadly. Physique (height, weight, and muscle tone) may represent important aspects of appearance. However, other aspects may also be included in overall appearance, such as size and shape of facial features, complexion, and so on. For example, Cunningham (1986) found that college men rated certain female facial features more attractive (e.g., large, round, widely separated eyes, a short nose, and a small chin) and suggested that these traits might signal youthfulness and fertility. Furthermore, people associate overall attractiveness with a variety of positive personality traits, including dominance, good social skills, happiness, and good mental health (Dion, Berscheid, \& Walster, 1972) as well as physical health (Grammer \& Thornhill, 1994). Overall physical appearance, therefore, may be associated with social anxiety because of societal stresses on overall attractiveness. Given the importance of features beyond physique in societal judgments of appearance, a measure that encompasses overall appearance beyond physique in the assessment of social anxiety regarding one's appearance may be useful.

We created the Social Appearance Anxiety Scale (SAAS) to assess fear of situations in which one's overall appearance, including but not limited to body shape, may be evaluated. The SAAS is a rationally derived measure, in which items were chosen based on examination of current measures of social anxiety, body image dissatisfaction, and body dysmorphic disorder. The format of the items was chosen to match general measures of social anxiety. Also the symptoms from the social anxiety disorder, body dysmorphic disorder, and eating disorder sections of the DSM-IV (American Psychiatric Association, 1994) were consulted during the generation of the SAAS item pool. Items were selected to tap into a general construct of anxiety about being evaluated for one's appearance, as opposed to anxiety about specific aspects of appearance (e.g., one's hair, one's nose, or one's chest size).

The current study sought to examine the psychometric properties of the SAAS, including factor structure, internal consistency, test-retest reliability, and convergent validity. Regarding convergent validity, the correlations between the SAAS and measures of social anxiety, body image dissatisfaction, and irrational core beliefs about the importance, meaning, and effects of appearance in one's life were of particular interest. Because social appearance anxiety may be a specific situational fear in social anxiety, it was predicted that the SAAS would be a subset of social anxiety. As a measure of concerns about social consequences 
of one's appearance, it was also predicted that the SAAS would be associated with other measures of appearancerelated concerns, such as the SPAS (Frederick \& Morrison, 1998; E. Hart et al., 1989) and measures of body image disturbance (e.g., Cash \& Labarge, 1996). Given previous research linking depression to higher SPAS scores (Diehl et al., 1998; Frederick \& Morrison, 1998), as well as higher social anxiety and body image disturbance (e.g., Cash \& Labarge, 1996; Cash \& Szymanski, 1995; Cash, Theriault, \& Annis, 2004), the association between scores on the SAAS and depression was examined. As a further check on the validity of the SAAS, the correlation with a measure of social desirability response bias was also examined, with a low correlation hypothesized to support the construct validity of the measure.

\section{METHOD}

\section{Procedure}

The study included three different samples of participants from two large public universities, who volunteered for the study in return for credit toward their research requirement for an introductory psychology course. Samples 1 and 3 were recruited at Temple University, whereas Sample 2 was recruited at the University of Buffalo (SUNY). Sample 1 provided the data for the Exploratory Factor Analysis (EFA), and Sample 2 provided the data for the Confirmatory Factor Analysis (CFA). To provide independent confirmation of the final factor structure, we conducted a second CFA using Sample 3. Data from participants who completed the additional packet of questionnaires were used to calculate the convergent validity of the SAAS, and a subsample of participants also completed retest of the SAAS to examine test-retest reliability. The convergent validity of the SAAS was examined with each of the measures of social anxiety and body image disturbance that were consulted in the initial creation of items for the SAAS.

\section{Participants}

Sample 1 comprised 512 participants (379 females, 132 males, and 1 did not report gender), with a mean age of $18.85(S D=3.15)$. Participants represented a wide range of ethnic/racial backgrounds (40.2\% White, $33.2 \%$ African American, 13.3\% Asian American, 9.9\% Latino, and 3.4\% Mixed or Other ethnic background). Sample 2 comprised 853 participants ( 385 females and 468 males), with a mean age of $19.34(S D=2.88)$. Sample 2 was $69.1 \%$ White, 9.4\% African American, 12.7\% Asian American, 4.6\%
Latino, and $4.3 \%$ Other or Mixed background (percentages add up to $100.1 \%$ because of rounding).

Sample 3 comprised 541 participants (376 females, 164 males, and 1 did not report gender), with a mean age of $19.01(S D=4.54)$. Regarding ethnic background, 38.5\% were White, $25.5 \%$ African American, $7.0 \%$ Asian American, 4.4\% Latino, and 24.6\% Mixed or Other ethnic background. One hundred fifty participants ( 97 females and 53 males) in Sample 3 volunteered to complete an additional packet of questionnaires containing the SAAS $(n=$ $110)$ or the SAAS alone $(n=40)$. Data from participants who completed the additional packet of questionnaires were used to calculate the convergent validity of the SAAS. Data from 100 participants (67 females and 33 males) were available to examine the test-retest reliability of the SAAS.

\section{Measures}

SAAS. The original version of the SAAS had 17 items. Participants indicate how characteristic each statement is on a Likert-type scale ranging from 1 (not at all) to 5 (extremely). Examples are "I get nervous talking to people because of the way I look," and "I worry that others talk about flaws in my appearance when I'm not around."

Brief Fear of Negative Evaluation (BFNE) Scale. The BFNE (Leary, 1983a) is a 12-item instrument that assesses concern with evaluation by others in social situations. Items are rated on a Likert-type scale ranging from 1 (not at all characteristic of me) to 5 (extremely characteristic of me). The measure demonstrated high internal consistency, $\alpha=$ .90 , and 4-week test-retest reliability, $r=.75$, in Leary's (1983a) undergraduate sample. Sample items are "I worry about what other people will think of me even when I know it doesn't make a difference," and "Sometimes I think I am too concerned with what other people think of me." The BFNE was also highly correlated with Watson and Friend's (1969) original 30-item true or false version of the scale $(r=.96$; Leary, 1983a). Rodebaugh et al. (2004) found this scale to be more sensitive than the Fear of Negative Evaluation Scale (FNE) to varying degrees of fear of negative evaluation in an item response theory analysis in a student sample. Weeks et al. (2005) reported that the BFNE was internally consistent and highly correlated with measures of social anxiety in a sample of patients with social anxiety disorder, and Collins, Westra, Dozois, and Stewart (2005) demonstrated that the BFNE discriminated patients with social anxiety disorder from patients with panic disorder. In the current study, the BFNE had an internal consistency of $\alpha=.79$.

SIAS and SPS. These measures (Mattick \& Clarke, 1998) are companion instruments, which assess social 
anxiety experienced in social interactions in dyads or groups, or when being scrutinized by others, respectively. Both scales contain 20 items rated on a Likert-type scale from 0 (not at all characteristic of me) to 4 (extremely characteristic of me). A sample SIAS item is "I have difficulty talking with other people." Both scales had excellent internal consistency in samples of patients with social anxiety disorder $(\alpha s \geq .89)$ and of college undergraduates ( $\alpha s \geq .88$; Mattick \& Clarke, 1998). Test-retest reliability for both scales was high $(r s \geq .91)$ in the social anxiety disorder sample. Both scales also have demonstrated good discriminant validity and convergent validity with other self-report measures of social anxiety (Brown et al., 1997; Heimberg, Mueller, Holt, Hope, \& Liebowitz, 1992). The SIAS was found to be more strongly correlated to other measures of social interaction anxiety and self-statements in social situations, whereas the SPS was more strongly related to measures of performance/observation fear. In the current study, internal consistencies were $\alpha=.85$ for the SIAS and $\alpha=.92$ for the SPS.

SPAS. The SPAS (E. Hart et al., 1989) is a 12-item measure that assesses the construct of social physique anxiety. E. Hart et al. defined social physique anxiety as anxiety that occurs as a result of the prospect or presence of interpersonal evaluation involving one's physique. The measure's focus is on physique-related issues such as body fat, muscle tone, and body proportions, and it does not include items assessing other domains of appearance-related anxiety. An example item is "It would make me uncomfortable to know others were evaluating my physique." Participants rate how characteristic each item is on a Likert-type scale ranging from 0 (not at all) to 4 (extremely). It demonstrated high internal consistency $(\alpha=.90)$ and 8 -week testretest reliability $(r=.82)$ in a sample of 89 university students (E. Hart et al., 1989). In a sample of 195 undergraduates, the measure was found to be weakly correlated with the Interaction Anxiousness Scale (Leary, 1983b; $r=.33)$ and the FNE Scale $(r=.35)$. Internal consistency in the current study was $\alpha=.90$.

Multidimensional Body-Self Relations QuestionnaireAppearance Scales (MBSRQ-AS). The MBSRQ-AS (Cash, $2000 \mathrm{~b}$ ) is a 34-item instrument that assesses body-image attitudes. Items are rated on a Likert-type scale ranging from 1 (definitely disagree) to 5 (definitely agree). This version is a shortened version of a widely used 69-item questionnaire (Cash, Winstead, \& Janda, 1986). Cash (2000b) asserts that subscales can be extracted from the overall measure without consequence to psychometric properties. Only the three scales associated with overall appearance were used in this study.
The seven items of the Appearance Evaluation subscale assess feelings of physical attractiveness or unattractiveness. Higher scores indicate greater positive feelings and satisfaction with his or her appearance. An example item is "I like my looks just the way I am." Internal consistency for this subscale was $\alpha=.88$ for both men and women. One-month test-retest reliability was $r=.81$ for men and $r=.91$ for women (Cash, 2000b). In the current study, these items had an internal consistency of $\alpha=.84$. The Appearance Orientation subscale has 12 items that examine the extent of investment in one's appearance. High scores indicate considerable emphasis on appearance and engagement in extensive grooming behaviors. Low scorers do not expend much effort on grooming behaviors and are generally apathetic about appearance. An example item is "Before going out in public, I always notice how I look." One-month test-retest reliability was $r=.89$ for men and $r=.90$ for women (Cash, 2000b). The Overweight Preoccupation subscale consists of four questions that assess anxiety about one's weight, weight vigilance, dieting, and eating restraint. An example item is "I constantly worry about being or becoming fat." This subscale had an $\alpha=.73$ for men and $\alpha=.76$ for women. One-month testretest reliability was $r=.79$ for men and $r=.89$ for women (Cash, 2000b). In the current study, the three subscales had internal consistencies of $\alpha=.84, .85$, and .81, respectively.

Appearance Schemas Inventory (ASI). The ASI (Cash \& Labarge, 1996) is a 14-item measure used to assess core beliefs about the importance, meaning, and effects of appearance on one's life. Participants rate each statement on a Likert-type scale ranging from 1 (strongly disagree) to 5 (strongly agree). Unlike other measures of body image, this measure was designed to assess dysfunctional body image schemas. For example, the measure asks women to rate "To be feminine, a woman must be as pretty as possible." The measure has high internal consistency, $\alpha=.84$, and 1-month test-retest reliability of $r=.72$ to .76 (Cash, 2000a). The ASI has been related to multiple aspects of body image but is not correlated with actual body mass (Cash \& Labarge, 1996). The internal consistency of this measure was $\alpha=.88$ in the current study.

Body-Image Ideals Questionnaire (BIQ). The BIQ (Cash \& Szymanski, 1995) measures self-ideal discrepancies and the influence of physical ideals on evaluation of physical attributes such as height, weight, muscularity, and complexion. Unlike other body image measures, this measure is based on self-discrepancy theory (Higgins, 1987), which when applied to body image, suggests that a person's body image satisfaction is based on the degree to which a person believes his or her physical characteristics 
match his or her internalized ideals and the importance associated with having those ideals (Cash \& Szymanski, 1995). For each statement about physical attributes, participants rate on a Likert-type scale how much they think they resemble their personal ideal of that attribute $(0=$ exactly as I am to $3=$ very unlike $m e$ ) and how important it is that they embody that ideal $(0=$ not important to $4=$ very important). BIQ scores are calculated by summing the arithmetic products of discrepancy and importance for each item. The 22-item questionnaire had $\alpha$ 's of .81 for men and .76 for women. For both men and women, scores correlated significantly with the Appearance Evaluation subscale of the MBSRQ $(r=-.65)$ and the ASI $(r=.55)$. In the current study, this scale had internal consistency of $\alpha=.88$.

Beck Depression Inventory (BDI). The BDI (Beck, Rush, Shaw, \& Emery, 1979) is a 21-item self-report measure of depression. Participants choose one of four responses to items concerned with various aspects of mood, cognition, and behavior as they relate to depression. It is a widely used measure of depression with an internal consistency of $\alpha=.88$ for nonpsychiatric populations (Beck, Steer, \& Garbin, 1988). It is highly correlated with the BDI-II (Beck, Steer, \& Brown, 1996) in both nonclinical and clinical samples $(r s=.93$; Beck et al., 1996; Dozois, Dobson, \& Ahnberg, 1998). In the current study, the internal consistency was $\alpha=.89$.

Marlowe-Crowne Social Desirability Scale-Short Form. This measure (Reynolds, 1982) has 13 items that assess one's tendency to present oneself in a socially desirable manner, using true or false responses, with higher scores indicating more of this tendency. This brief form was derived from the original 33-item instrument (Crowne \& Marlowe, 1960). Reynolds (1982) found that the two measures were highly correlated $(r=.93)$ and recommends the use of the shorter version to more easily assess socially desirable response tendencies in research that uses self-report measures. Internal consistency in the present study was $\alpha=.71$.

\section{RESULTS}

\section{Factor Structure}

Statistical methodology. Traditional Exploratory and Confirmatory Factor Analytic methods based on Pearson product-moment correlations (or covariances) assume continuous, normally distributed variables, whereas Likerttype items elicit variables with categorical, ordinal distributions (see Nunnally \& Bernstein, 1994; West, Finch, \&
Curran, 1995). As a result, factor analyses based on productmoment relations produce biased tests of model fit, as well as biased factor loadings and standard errors (for reviews see Flora \& Curran, 2004; West et al., 1995). Therefore, we examined the factorial structure of the SAAS using methods that explicitly account for the ordinal nature of the item response variables. In particular, we fitted each factor model to a matrix of polychoric correlations using weighted-least squares (WLS) estimation (Muthén, 1984, 1993). Simulation work has shown that this methodology performs well when ordinal variables are factor analyzed using sample sizes in the range available here (Flora \& Curran, 2004). Other applications using this method include Flora, Finkel, and Foshee (2003) and Rodebaugh et al. (2004). In the current analyses, all factor models were estimated using Mplus (version 3.12; Muthén \& Muthén, 2005).

$E P A$. A scree plot of eigenvalues from the polychoric correlation matrix was strongly indicative of a one-factor solution for the set of 17 items from the SAAS (see Figure 1). In addition, the root mean square error of approximation (RMSEA; Steiger, 1990) model fit index was .069 , indicating reasonable fit of the one-factor model to the data (Browne \& Cudeck, 1993). ${ }^{1}$ All items had factor loadings of at least |.75|, except one, which had a loading of .35 (corresponding to a communality estimate of only .12 with unexplained variance of .88). This item "I rarely get tense when I think others are evaluating my looks" was dropped from all subsequent analyses.

CFA. Because the $\chi^{2}$ fit statistic is typically inflated when confirmatory models are fit to polychoric correlations using WLS estimation (e.g., Flora \& Curran, 2004), we examined additional fit indices to judge the fit of the unidimensional model suggested by the exploratory analysis. The RSMEA statistic has been shown to perform well with this ordinal variable methodology (Hutchinson \& Olmos, 1998). It has also been suggested that the general guidelines of $\mathrm{Hu}$ and Bentler (1999) for commonly used fit indices are appropriate (Yu \& Muthén, 2001, as cited in Muthén \& Muthén, 2005). These guidelines suggest that a model of good fit has a Tucker Lewis Index (TLI; Tucker \& Lewis, 1973) or Comparative Fit Index (CFI; Bentler, $1990) \geq .95$ combined with an RMSEA close to .06 .

A one-factor model for the 16 SAAS items fits the Sample 2 data well, $\chi^{2}(104, N=853)=381.21 ; p<.001$; RMSEA $=.056 ; \mathrm{CFI}=.99 ; \mathrm{TLI}=.99$. In addition, each item was associated with a large and statistically significant factor loading (all $p s<.001$ ). This one-factor model also fits the Sample 3 data well, $\chi^{2}(104, N=541)=$ 311.89; $p<.001$; RMSEA $=.061 ; \mathrm{CFI}=.99 ; \mathrm{TLI}=.99$, again with each item showing a strong factor loading. 
FIGURE 1

Scree Plot of Eigenvalues

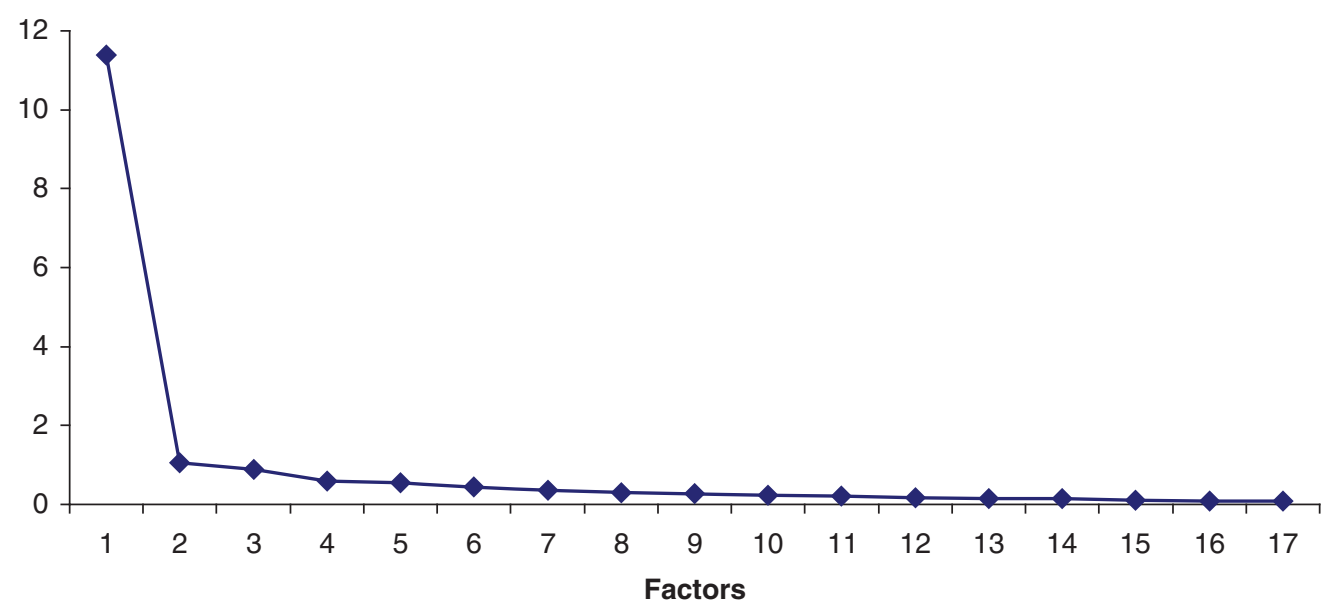

TABLE 1

Factor Loadings for the 16-Item Social Appearance Anxiety Scale in Samples 2 and 3

\begin{tabular}{lcc}
\hline & Factor Loading \\
\cline { 2 - 3 } Item & Sample 2 \\
\hline 1. I feel comfortable with the way I appear to others.* & .67 & Sample 3 \\
2. I feel nervous when having my picture taken. & .66 & .78 \\
3. I get tense when it is obvious people are looking at me. & .95 & .97 \\
4. I am concerned people would not like me because of the way I look. & .91 \\
5. I worry that others talk about flaws in my appearance when I am not around. & .96 \\
6. I am concerned people will find me unappealing because of my appearance. & .98 & .97 \\
7. I am afraid that people find me unattractive. & .97 \\
8. I worry that my appearance will make life more difficult for me. & .94 \\
9. I am concerned that I have missed out on opportunities because of my appearance. & .91 \\
10. I get nervous when talking to people because of the way I look. & .96 \\
11. I feel anxious when other people say something about my appearance. & .98 \\
12. I am frequently afraid I would not meet others' standards of how I should look. & .91 \\
13. I worry people will judge the way I look negatively. & .91 \\
14. I am uncomfortable when I think others are noticing flaws in my appearance. & .96 \\
15. I worry that a romantic partner will/would leave me because of my appearance. & .96 \\
16. I am concerned that people think I am not good looking. & .99 \\
\hline
\end{tabular}

NOTE: $N=853$ (Sample 2); $N=541$ (Sample 3). *Item 1 is reverse-coded.

The factor loadings from both samples are given in Table 1. Based on these CFA results, combined with the EFA findings, there appears to be sufficient evidence to treat the 16-item SAAS as a unidimensional measure.

\section{Internal Consistency and Test-Retest Reliability}

The 16-item SAAS demonstrated good internal consistency in all three samples, $\alpha \mathrm{s}=.94, .95$, and .94 , respectively.
We also calculated 1-month test-retest reliability with a subset of 100 participants from Sample 3. Mean time between administrations was 32.76 days $(S D=6.86$; range 21-42). The SAAS demonstrated good test-retest reliability, $r=.84$.

\section{Convergent and Discriminant Validity}

We first investigated the convergent validity of the SAAS in a subsample of students from Sample 3. Because of the 
TABLE 2

Zero-Order Correlations Between the Social Appearance Anxiety Scale 16-Item Total Score and Measures of Social Anxiety, Body Image Dissatisfaction, and Related Constructs

\begin{tabular}{|c|c|c|c|c|c|c|c|c|c|c|c|c|c|}
\hline & $S A A S$ & $B F N E$ & SIAS & $S P S$ & $A S I$ & $B I Q$ & AppEval & OwPreoc & $S P A S$ & AppOr & $B D I$ & Sex & $S D S$ \\
\hline SAAS & - & & & & & & & & & & & & \\
\hline BFNE & $.82 *$ & - & & & & & & & & & & & \\
\hline SIAS & $.76^{*}$ & $.71 *$ & - & & & & & & & & & & \\
\hline SPS & $.78^{*}$ & $.68^{*}$ & $.80^{*}$ & - & & & & & & & & & \\
\hline ASI & $.49 *$ & $.53 *$ & $.32 *$ & $.45^{*}$ & - & & & & & & & & \\
\hline BIQ & $.58 *$ & $.63 *$ & $.48 *$ & $.40 *$ & $.59 *$ & - & & & & & & & \\
\hline AppEval & $-.55^{*}$ & $-.51 *$ & $-.46^{*}$ & $-.42 *$ & $-.37 *$ & $-.54 *$ & - & & & & & & \\
\hline OwPreoc & $.38^{*}$ & $.39 *$ & $.32 *$ & $.39 *$ & $.42 *$ & $.48 *$ & -.45 & - & & & & & \\
\hline SPAS & $.59 *$ & $.61 *$ & $.46^{*}$ & $.46^{*}$ & $.50 *$ & $.63^{*}$ & -.78 & $.60 *$ & - & & & & \\
\hline AppOr & .23 & $.34 *$ & .04 & .13 & $.47 *$ & $.34 *$ & -.01 & $.47 *$ & .26 & - & & & \\
\hline BDI & $.52 *$ & $.40^{*}$ & $.42 *$ & $.44 *$ & $.28 *$ & $.40 *$ & -.48 & $.28 *$ & $.46^{*}$ & -.001 & - & & \\
\hline Sex & -.11 & -.08 & .03 & .01 & -.03 & -.06 & .16 & -.13 & $-.31 *$ & -.10 & -.12 & - & \\
\hline SDS & -.20 & -.25 & -.26 & -.25 & -.09 & -.17 & .16 & -.18 & -.24 & -.02 & $-.37 *$ & -.16 & - \\
\hline
\end{tabular}

NOTE: SAAS = Social Appearance Anxiety Scale; BFNE = Brief Fear of Negative Evaluation Scale; SIAS = Social Interaction Anxiety Scale; SPS = Social Phobia Scale; ASI = Appearance Schemas Inventory; BIQ = Body Image Ideals Questionnaire; AppEval = Multidimensional Body-Self Relations Questionnaire-Appearance Evaluation subscale; OwPreoc $=$ Multidimensional Body-Self Relations Questionnaire Overweight Preoccupation scale; SPAS = Social Physique Anxiety Scale; AppOr = Multidimensional Body-Self Relations Questionnaire-Appearance Orientation scale; BDI $=$ Beck Depression Inventory; Sex $=$ biological sex $(0=$ female, $1=$ male $)$. SDS $=$ Social Desirability Scale. High scores on the Appearance Evaluation scale indicate the participant feels mostly positive and satisfied with his or her appearance, whereas low scores indicate unhappiness and dissatisfaction with physical appearance.

$* p<.005$.

large number of correlations, we applied a Bonferroni correction, resulting in a critical significance level of .005 . Pearson product-moment correlations are presented in Table 2. The SAAS was significantly positively correlated with measures of social anxiety. The SAAS was significantly positively correlated with the SPAS, as well as with measures of body image dissatisfaction. The SAAS's significant negative correlation with the Appearance Evaluation subscale indicates that higher scores on the SAAS were related to feelings of unattractiveness. The Overweight Preoccupation subscale was positively correlated with scores on the SAAS, suggesting that eating restraint, weight vigilance, fat anxiety, and dieting are associated with social appearance anxiety. SAAS scores were not associated with female versus male participants. Finally, the SAAS was positively related to the BDI but not with the Marlowe-Crowne Social Desirability Scale-Short Form.

To further examine the relationships between the SAAS and related measures, we specified a structural equation model (SEM) consisting of two correlated latent factors: A Social Anxiety factor that was measured by the BFNE, SIAS, SPAS, and SPS, and a Body Image factor measured by the BIQ, ASI, and the three subscales of the MBSRQ. The SAAS was allowed to correlate with these two factors to determine its relationship with social anxiety and body image. We estimated the structural model using maximumlikelihood with the Satorra-Bentler scaled chi-square and robust standard error estimation (Satorra \& Bentler, 1988).

The initial model did not fit the data acceptably, $\chi^{2}(33)$ $=162.52 ; p<.001 ;$ with RMSEA $=.19 ; \mathrm{CFI}=.78 ;$ and $\mathrm{TLI}=.70$. This poor model fit was mostly attributable to the fact that the Appearance Orientation subscale of the MBSRQ had a weak correlation with the Appearance Evaluation subscale $(r=-.11)$, despite having moderate correlations with all other measures on the Body Image factor. Therefore, we modified the structural model so that the Appearance Orientation subscale was no longer an indicator of the Body Image factor, but was still allowed to correlate with the SAAS and other measures of body image, except Appearance Evaluation. Conceptually, by removing the Appearance Orientation subscale from the Body Image factor, the Body Image factor more appropriately measures only negative body image, as opposed to the combination of body image and focus on appearance.

The revised model was somewhat better but still did not fit the data adequately, $\chi^{2}(29)=117.94 ; p<.001 ;$ RMSEA $=.17 ; \mathrm{CFI}=.85 ; \mathrm{TLI}=.77$. Next, we noted a large modification index regarding the relationship between the SPAS and the negative Body Image factor; thus, we included the SPAS as an indicator of body image as opposed to social anxiety. Although the SPAS has been conceptualized variously as either a measure of social anxiety (Frederick \& Morrison, 1998; E. Hart et al., 
FIGURE 2

\section{Structural Model Demonstrating the Relationship of the Social Appearance Anxiety Scale to Measures of Social Anxiety and Negative Body Image.}

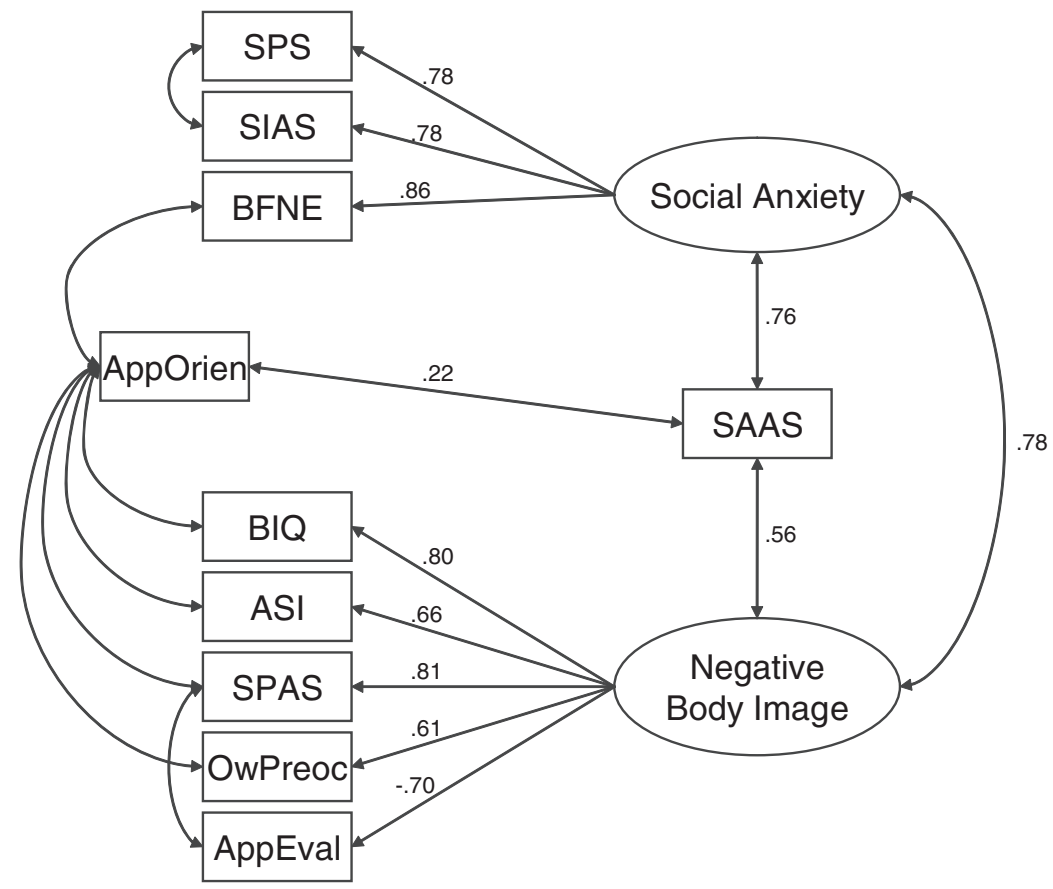

NOTE: All paths are significant $(p<.05)$. SAAS $=$ Social Appearance Anxiety Scale; Brief Fear of Negative Evaluation $=$ Brief Fear of Negative Evaluation Scale; Social Interaction Anxiety Scale = Social Interaction Anxiety Scale; Social Phobia Scale = Social Phobia Scale; Appearance Schemas Inventory = Appearance Schemas Inventory; Body Image Ideals Questionnaire = Body Image Ideals Questionnaire; AppEval = Multidimensional Body-Self Relations Questionnaire - Appearance Evaluation Subscale; OwPreoc = Multidimensional Body-Self Relations Questionnaire - Overweight Preoccupation Scale; Social Physique Anxiety Scale = Social Physique Anxiety Scale; AppOrien = Multidimensional Body-Self Relations Questionnaire - Appearance Orientation Scale. High scores on the Appearance Evaluation Scale indicate the participant feels mostly positive and satisfied with his/her appearance, while low scores indicate unhappiness and dissatisfaction with physical appearance.

1989) or a measure of body image (Davison \& McCabe, 2006; Ginis, Eng, Arbour, Hartman, \& Phillips, 2005), none of these studies examined structural models of the SPAS, which assesses feared consequences related to one's body shape. In addition, we allowed certain correlations among residual variance terms as suggested by modification indices. ${ }^{2}$ Ordinarily this type of data-driven model modification should be avoided. However, here our sole purpose is to examine the role of the SAAS in the context of these related measures, and none of these added error covariance terms affects the interpretation of the relationships between the SAAS and the social anxiety and negative Body Image factors. This final model had good fit, $\chi^{2}(33)=47.32 ; p=.05 ; \mathrm{RMSEA}=.06 ; \mathrm{CFI}=$ .98 ; TLI $=.96$. A path diagram of this model is given in Figure 2.

A key result from this SEM analysis is that the SAAS was significantly correlated with both the Social Anxiety factor $(r=.76 ; p<.001)$ and the negative Body Image factor $(r=.56 ; p<.001)$. This finding implies that the
SAAS has good convergent validity with respect to both other measures related to social anxiety and measures related to body image disturbance. This finding was expected because the SAAS was created to be a link between Social Anxiety and negative Body Image that was not specific to physique concerns but appearance more broadly defined.

Given the SEM findings described above, it was next of interest to explore the incremental validity of the SAAS with respect to the measurement of Social Anxiety and negative Body Image. First, a canonical correlation analysis was conducted using the measures of social anxiety as outcomes, and measures of negative body image and the SAAS were regressors. The first canonical correlation accounted for $79 \%$ of the shared variance between these regressors and outcomes, with the SAAS having the largest standardized canonical coefficient (.86) of all regressors (the SPAS had the second highest coefficient, which equaled .10). Following up this analysis, controlling for the negative body image variables, the SAAS had 
a significant squared semipartial correlations of $.34(p<$ $.0001)$ with the SPS, $.31(p<.0001)$ with the SIAS, and $.22(p<.0001)$ with the BFNE. Therefore, the SAAS explained a unique proportion of variability in social anxiety, above and beyond negative body image.

Another canonical correlation analysis was conducted using the measures of negative body image as outcomes, with measures of social and the SAAS as regressors. The first canonical correlation accounted for 53\% of the shared variance between these regressors and outcomes, with the SAAS having the second largest standardized canonical coefficient (.43) of all regressors (the BFNE had the highest coefficient, which equaled .70). Controlling for the social anxiety variables, the SAAS had significant squared semipartial correlations of $.04(p=.02)$ with the Appearance Evaluation subscale and $.02(p=.05)$ with the SPAS. Thus, there was some evidence that the SAAS was a unique predictor of negative body image over and above social anxiety.

To examine the predictive validity of the SAAS with respect to depression, we estimated a multiple regression model to determine whether the SAAS was a significant predictor of BDI scores above and beyond the measures of social anxiety. Controlling for scores on the BFNE, SIAS, and SPS, the SAAS was a significant predictor of depression, $p=.02$, with a squared semipartial correlation of .04. Next, we estimated another regression model to determine whether the SAAS predicted BDI scores when controlling for the measures of negative body image. Controlling for scores on the BIQ, ASI, SPAS, and the Overweight Preoccupation and Appearance Evaluation scales, the SAAS was again significantly related to depression, $p<$ .01 , with a squared semipartial correlation of .06. Thus, this analysis provides evidence for the predictive and incremental validity of the SAAS.

\section{DISCUSSION}

The development of the SAAS addressed the need for a measure of anxiety in situations where one's appearance may be evaluated. This study examined the SAAS in 3 samples, resulting in a 16-item unidimensional solution supported by good fit indices in two confirmatory factor analyses. The 16-item SAAS demonstrated high internal consistency and convergent validity with other measures of social anxiety and body image disturbance. The test-retest reliability of the SAAS suggests that social appearance anxiety is stable across time and that the SAAS may be a reliable measure of this construct over a period of time.
In bivariate relationships, the SAAS was highly correlated with measures of social anxiety and moderately associated with the SPAS. Thus, social appearance anxiety appears to fall within the larger class of social anxiety and is also moderately, but not strongly, related to a similar domain of fear, namely, social physique anxiety. In contrast to previous research not using structural modeling that discussed the SPAS as a measure of social anxiety (Frederick \& Morrison, 1998; Hart et al., 1989), findings from the structural model suggest that the SPAS may be better thought of as a subset of body image disturbance.

The SAAS also appears to be an indicator of negative body image. Higher scores on the SAAS related to greater disparity between self-reported actual and ideal physical attributes, dysfunctional schemas about the importance and meaning of appearance, feelings of unattractiveness, emphasis on appearance and its maintenance, and a preoccupation with being or becoming overweight. Given the associations between social anxiety and maladaptive appearance schemas, and body image disturbance in both normative and clinical samples (e.g., Cash \& Labarge, 1996; Hinrichsen et al., 2004), there may be some utility in having a measure that taps into both social anxiety and negative body image that encompasses but is not limited to physique. It should be noted that coefficients were typically high with social anxiety measures but more moderate with body image disturbance measures. It is therefore possible that the social appearance anxiety would be just as, if not more, important in the etiology and/or maintenance of social anxiety as it might be in body image disturbance.

SAAS scores were only marginally associated with investment in one's grooming. This was not surprising as many of the items in the Appearance Orientation subscale, such as "Before going out, I usually spend a lot of time getting ready," and "I take special care with my hair grooming" are not necessarily associated with social anxiety. In fact, many people may take great pride in their grooming, which would not necessarily be consistent with high scores on a measure such as the SAAS. The lack of correlation between the SAAS and social desirability response bias suggests that lower scores on the SAAS were not attributable to participants attempting to present themselves in a socially desirable fashion.

Regarding predictive validity, SAAS appeared to be a unique predictor of depression, above and beyond the social anxiety indicators and the negative body image indicators. In addition, although evidence suggests that the SAAS is highly related to social anxiety, the SAAS also appears related to measures of body image dissatisfaction. The SAAS also appears to tap into previously unexplored 
areas between social anxiety and body image disturbance, explaining a unique proportion of variability in social anxiety beyond negative body image, and to a more limited extent, unique variability in body image beyond more general measures of social anxiety. In addition, this study evaluated the sensitivity of the SAAS to appearance-related concerns, but future research needs to demonstrate that it is a specific measure of this situational domain. Although the SAAS was not associated with frequent self-grooming, it is unknown to what extent social appearance anxiety would affect specific behaviors associated with appearance- related image disturbance, such as compulsive exercising, changing one's food intake, or seeking cosmetic surgery. The convergent and predictive validity of the SAAS can be further established by examining other outcome measures related to social anxiety, such as general anxiety, self-esteem, and social self-efficacies in interpersonal and situations.

Because perspectives on appearance are affected by gender and cultural differences (e.g., Russell \& Cox, 2003), future studies may also include these variables in the psychometric analysis of the SAAS. The effects of social appearance anxiety may be more related to drive for muscularity and anabolic steroid use among males (e.g., Barnett, Keel, \& Conoscenti, 2001; see Cafri \& Thompson, 2004, for a review) versus drive for thinness or food restriction among females (e.g., Barnett et al., 2001; Diehl et al., 1998). Gender may interact with cultural differences in predicting social appearance anxiety, as one study suggested that Asian American and not White American men perceived themselves to be too thin (Barnett et al., 2001).

Given that the psychometric properties of the SAAS were examined in a normative sample of undergraduate students, future work needs to further examine the use of this measure with clinical populations, such as social anxiety disorder, eating disorders, or body dysmorphic disorder, and people with medical conditions affecting their appearance (e.g., patients with dermatological conditions or patients who have had disfiguring surgeries), as well as to examine associations with depression in these clinical samples. It should be noted that given the specific nature of many complaints for body dysmorphic disorder, a more specific scale than the SAAS, measuring social appearance anxiety regarding specific appearance features may be useful for this population. Once the psychometric properties of the SAAS are established in clinical samples, examination of the role of social appearance anxiety in the etiology and maintenance of social anxiety and/or body image would be warranted. More research is needed to further examine the discriminant validity of the SAAS, as well as confirm the factor structure of the 16-item measure in other nonclinical samples (e.g., high school students, older adult samples).
Future work may also wish to examine associations between the SAAS and another recently developed measure, the Fear of Negative Appearance Evaluation Scale (FNAES; Lundgren, Anderson, \& Thompson, 2004). The FNAES was related to both the original Fear of Negative Evaluation Scale (Watson \& Friend, 1969) and body image disturbance scales among female undergraduate students. It is unknown to what extent this measure is similar to the SAAS or whether the FNAES is an indicator of social anxiety versus body image disturbance.

The 16-item SAAS appears to be a psychometrically valid measure of social anxiety about one's appearance. The SAAS correlates positively both with previous measures of social anxiety and body image dissatisfaction, and it demonstrates high test-retest reliability and internal consistency. This measure may be useful in the assessment of appearance-related social anxiety in normal populations and perhaps with clinical populations as well, pending further research.

\section{NOTES}

1. A two-factor solution with Promax rotation, which allows factors to be correlated, was also examined. Although this model had a slightly lower RMSEA (.060), the second factor was defined primarily by only two items and the interfactor correlation was .61. Thus, the one-factor solution was retained.

2. Specifically, we freed correlations if the associated expected parameter change was greater than .20 in the correlation metric. Most modification indices suggested a parameter change of less than .10, and there were only four error correlations associated with a change of .20 or above.

\section{REFERENCES}

American Psychiatric Association. (1994). Diagnostic and statistical manual of mental disorders (4th ed.). Washington, DC: Author.

American Psychiatric Association. (2000). Diagnostic and statistical manual of mental disorders (4th ed., text revision). Washington, DC: Author.

Barnett, H. L., Keel, P. K., \& Conoscenti, L. M. (2001). Body type preferences in Asian and Caucasian college students. Sex Roles, 45, 867-878.

Beck, A. T., Rush, A. J., Shaw, B. F., \& Emery, G. (1979). Cognitive therapy of depression. New York: Guilford.

Beck, A. T., Steer, R. A., \& Brown, G. K. (1996). Beck Depression Inventory manual (2nd ed.). San Antonio, TX: The Psychological Corporation.

Beck, A. T., Steer, R. E., \& Garbin, M. G. (1988). Psychometric properties of the Beck Depression Inventory: Twenty-five years of evaluation. Clinical Psychology Review, 8, 77-100.

Bentler, P. M. (1990). Comparative fix indexes in structural models. Psychological Bulletin, 107, 238-246.

Brown, E. J., Turovsky, J., Heimberg, R. G., Juster, H. R., Brown, T. A., \& Barlow, D. H. (1997). Validation of the Social Interaction Anxiety Scale and the Social Phobia Scale across the anxiety disorders. Psychological Assessment, 9, 21-27. 
Browne, M. W., \& Cudeck, R. (1993). Alternative ways of assessing model fit. In K. A. Bollen \& J. S. Long (Eds.), Testing structural equation models (pp. 136-162). Newbury Park, CA: Sage.

Cafri, G., \& Thompson, J. K. (2004). Evaluating the convergence of muscle appearance attitude measures. Assessment, 11, 224-229.

Cash, T. F. (2000a). Users manual for the Appearance Schemas Inventory. Unpublished manuscript, Old Dominion University, Norfolk, VA.

Cash, T. F. (2000b). Users manual for the Multidimensional Body-Self Relations Questionnaire. Unpublished manuscript, Old Dominion University, Norfolk, VA.

Cash, T. F., \& Labarge, A. S. (1996). Development of the Appearance Schemas Inventory: A new cognitive body-image assessment. Cognitive Therapy and Research, 20, 37-50.

Cash, T. F., \& Szymanski, M. L. (1995). The development and validation of the Body-Image Ideals Questionnaire. Journal of Personality Assessment, 64, 466-477.

Cash, T. F., Theriault, J., \& Annis, N. M. (2004). Body image in an interpersonal context: Adult attachment, fear of intimacy, and social anxiety. Journal of Social and Clinical Psychology, 23, 89-103.

Cash, T. F., Winstead, B. W., \& Janda, L. H. (1986). The great American shape-up: Body image survey report. Psychology Today, 20(4), 30-37.

Coles, M. E., Phillips, K. A., Menard, W., Pagano, M. E., Fay, C., Weisberg, R. B., et al. (2006). Body dysmorphic disorder and social phobia: cross-sectional and prospective data. Depression and Anxiety, $23,26-33$

Collins, K. A., Westra, H. A., Dozois, D. J. A., \& Stewart, S. H. (2005). The validity of the brief version of the Fear of Negative Evaluation Scale. Journal of Anxiety Disorders, 19, 345-359.

Crowne, D. P., \& Marlowe, D. (1960). A new scale of social desirability independent of psychopathology. Journal of Consulting Psychology, $24,349-354$.

Cunningham, M. R. (1986). Measuring the physical attractiveness: Quasi-experiments on the sociobiology of female facial beauty. Journal of Personality and Social Psychology, 50, 925-935.

Davison, T. E., \& McCabe, M. P. (2006). Adolescent body image and psychosocial functioning. Journal of Social Psychology, $146,15-30$

Diehl, N. S., Johnson, C. E., Rogers, R. L., \& Petrie, T. A. (1998). Social physique anxiety and disordered eating: What's the connection? Addictive Behaviors, 23, 1-6.

Dion, K., Berscheid, E., \& Walster, E. (1972). What is beautiful is good. Journal of Personality and Social Psychology, 24, 285-290.

Dozois, D. J. A., Dobson, K. S., \& Ahnberg, J. L. (1998). A psychometric evaluation of the Beck Depression Inventory-II. Psychological Assessment, 10, 83-89.

Flora, D. B., \& Curran, P. J. (2004). An evaluation of alternative methods for confirmatory factor analysis with ordinal data. Psychological Methods, 9, 466-491.

Flora, D. B., Finkel, E., \& Foshee, V. (2003). Higher order factor structure of a self-control test: Evidence from confirmatory factor analysis with polychoric correlations. Educational and Psychological Measurement, 63, 112-127.

Frederick, C. M., \& Morrison, C. S. (1998). A mediational model of social physique anxiety and eating disordered behaviors. Perceptual and Motor Skills, 86, 139-145.

Ginis, K. A. M., Eng, J. J., Arbour, K. P., Hartman, J. W., \& Phillips, S. M. (2005). Mind over muscle? Sex differences in the relationship between body image change and subjective and objective physical changes following a 12-week strength-training program. Body Image, 2, 362-272.

Grammer, K., \& Thornhill, R. (1994). Human (Homo sapiens) facial attractiveness and sexual selection: The role of symmetry and averageness. Journal of Comparative Psychology, 108, 233-242.

Hart, E., Leary, M., \& Rejeski, W. J. (1989). The measurement of social physique anxiety. Journal of Sport and Exercise Psychology, 11, 94-104
Heimberg, R. G., Mueller, G. P., Holt, C. S., Hope, D. A., \& Liebowitz, M. R. (1992). Assessment of anxiety in the social interaction and being observed by others: The Social Interaction Anxiety Scale and the Social Phobia Scale. Behavior Therapy, 23, 53-73.

Higgins, E. T. (1987). Self-discrepancy: A theory relating self and affect. Psychological Review, 94, 319-340.

Hinrichsen, H., Waller, G., \& van Gerko, K. (2004). Social anxiety and agoraphobia in the eating disorders: Associations with eating attitudes and behaviours. Eating Behaviors, 5, 285-290.

Holt, C. S., Heimberg, R. G., Hope, D. A., \& Liebowitz, M. R. (1992). Situational domains of social phobia. Journal of Anxiety Disorders, 6, 63-77.

Hu, L., \& Bentler, P. M. (1999). Cutoff criteria for fit indexes in covariance structure analysis: Conventional criteria versus new alternatives. Structural Equation Modeling, 6, 1-55.

Hutchinson, S. R., \& Olmos, A. (1998). Behavior of descriptive fit indexes in confirmatory factor analysis using ordered categorical data. Structural Equation Modeling, 5, 344-364.

Leary, M. R. (1983a). A brief version of the Fear of Negative Evaluation Scale. Personality and Social Psychology Bulletin, 9, 371-375.

Leary, M. R. (1983b). Social anxiousness: The construct and its measurement. Journal of Personality Assessment, 47, 66-75.

Liebowitz, M. R. (1987). Social phobia. Modern Problems in Pharmacopsychiatry, 22, 141-173.

Lundgren, J. D., Anderson, D. A., \& Thompson, J. K. (2004). Fear of negative appearance evaluation: Development and evaluation of a new construct for risk factor work in the field of eating disorders. Eating Behaviors, 5, 75-84.

Mattick, R. P., \& Clarke, J. C. (1998). Development and validation of measures of social phobia scrutiny fear and social interaction anxiety. Behaviour Research and Therapy, 36, 455-470.

Muthén, B. (1984). A general structural equation model with dichotomous, ordered categorical, and continuous latent variable indicators. Psychometrika, 49, 115-132.

Muthén, B. (1993). Goodness of fit with categorical and other nonnormal variables. In K. A. Bollen \& J. S. Long (Eds.), Testing structural equation models (pp. 205-234). Newbury Park, CA: Sage.

Muthén, L. K., \& Muthén, B. (2005). Mplus user's guide. Los Angeles: Author.

Nunnally, J. C., \& Bernstein, I. H. (1994). Psychometric theory (3rd ed.). New York: McGraw-Hill.

Pinto, A., \& Phillips, K. A. (2005). Social anxiety in body dysmorphic disorder. Body Image, 2, 401-405.

Reynolds, W. M. (1982). Development of reliable and valid short forms of the Marlowe-Crowne Social Desirability Scale. Journal of Clinical Psychology, 38, 119-125.

Rodebaugh, T. L., Woods, C. M., Thissen, D. M., Heimberg, R. G., Chambless, D. L., \& Rapee, R. M. (2004). More information from fewer questions: The factor structure and item properties of the original and Brief Fear of Negative Evaluation Scale. Psychological Assessment, 16, 169-181.

Russell, W. D., \& Cox, R. H. (2003). Social physique anxiety, body dissatisfaction, and self-esteem in college females of differing exercise frequency, perceived weight discrepancy, and race. Journal of Sport Behavior, 26, 297-318.

Safren, S. A., Heimberg, R. G., Brown, E. J., \& Holle, C. (1997). Quality of life in social phobia. Depression and Anxiety, 4, 126-133.

Satorra, A., \& Bentler, P. M. (1988). Scaling corrections for chi-square statistics in covariance structure analysis. 1988 ASA Proceedings of the Business and Economic Statistics Section (pp. 308-313). Alexandria, VA: American Statistical Association.

Schneier, F. R., Heckelman, L. R., Garfinkel, R., Campeas, R., Fallon, B. A., Gitow, A., et al. (1994). Functional impairment in social phobia. Journal of Clinical Psychiatry, 55, 322-331.

Steiger, J. H. (1990). Structural model evaluation and modification: An interval estimation approach. Multivariate Behavioral Research, 25, 173-180. 
Tucker, L. R., \& Lewis, C. (1973). A reliability coefficient for maximum likelihood factor analysis. Psychometrika, 38, 1-10.

Turner, S. M., Beidel, D. C., Dancu, C. V., \& Stanley, M. A. (1989). An empirically derived inventory to measure social fears and anxiety: The Social Phobia and Anxiety Inventory. Psychological Assessment, 1, 35-40.

Watson, D., \& Friend, R. (1969). Measurement of social-evaluative anxiety. Journal of Consulting and Clinical Psychology, 33, 448-457.
Weeks, J. W., Heimberg, R. G., Fresco, D. M., Hart, T. A., Turk, C. L., Schneier, F. R., et al. (2005). Empirical validation and psychometric evaluation of the Brief Fear of Negative Evaluation Scale in patients with social anxiety disorder. Psychological Assessment, 17, 179-190.

West, S. G., Finch, J. F., \& Curran, P. J. (1995). Structural equation models with nonnormal variables: Problems and remedies. In R. H. Hoyle (Ed.), Structural equation modeling (pp. 56-75). Thousand Oaks, CA: Sage. 